\title{
Improvement of Ant Colony Adhoc On-Demand Vector (ANT-AODV) with K-Means Clustering Nodes Method in Mobile Adhoc Network (MANET) Environment
}

\author{
Dimas W. L. Pamungkas ${ }^{(1)}$, Radityo Anggoro( ${ }^{(2)}$ \\ ${ }^{(1)(2)}$ Department of Informatics, Institut Teknologi Sepuluh Nopember, Indonesia \\ E-mail: ${ }^{(1)}$ dimaswlp@ce.its.ac.id; ${ }^{(2)}$ onggo@if.its.ac.id
}

Received: 15 December 2020; Revised: 24 April 2021; Accepted: 5 May 2021

\begin{abstract}
MANET (Mobile Ad hoc Network) is a technology used for data communication on mobile media. MANET moves and speeds also randomly with different distances between nodes. In the AODV reactive protocol, the development of a routing protocol with an ant colony model can significantly improve performance. Common problems such as energy problems, data congestion, distance to the signal strength of the wireless network frequency need to be measured and trying to solve. The measurement results will impact the decision on the development of the ant colony protocol implemented in AODV to obtain the best route for data transmission. Several studies have developed the K-Means clustering method to obtain the shortest and best route from the AODV protocol. It does not measure congestion, distance, energy, and signal strength of the wireless network. In this research, a combination of methods was carried out, by combining clustering and also ant colony method to get the best route while dealing with the problems that have been mentioned. Implementation is done by modifying the AODV protocol and adding the two methods. At the end of the research, metric measurements were carried out to determine the Packet Delivery Ratio, End to End Delay and also the Average Hop Count. The increase of the QOS value can be measured from the success of increasing the Packet Delivery Ratio, as well as decreasing the entire value of Delay and also the Average Hop Count. Simulations in this research were tried with the Random Way Point method according to the environment referring to related literature. From the simulation, it can be seen that the improvement of Quality of Service (QoS) on the modified AODV protocol with these two methods.
\end{abstract}

Keywords: manet, aodv, ant colony, k-means, clustering, wireless, network

\section{INTRODUCTION}

MANET network is a special network that works in a multi-hop peer-to-peer manner in which every information packet is sent from the source node to the destination via an intermediate node. MANET network can operate independently or with several routers connected to the internet network. The topology of the MANET network is dynamic, where each node can be joined or separated from the network anytime and anywhere. Singh \& Kumar (2017) Us- ing Mobile Ad-hoc network must be able to be used ad hoc, which is activated quickly and can independently manage data communication routing (Sharma \& Kumar, 2016).

Research related to the development of the AODV protocol on MANET by utilizing ant colony modifications in 2009 has been carried out. Wang, Osagie, Thulasiraman, \& Thulasiram (2009) Utilizing pheromones for the metric measurement into consideration of the selected pathway is a hallmark of ant colony use. Research related to Ant-Colony in 2015 was also 
carried out in research on the Dynamic Source Routing (DSR) reactive protocol.(Chatterjee \& Das, 2015) This research proposed a reactive routing protocol that combines Ant Colony (ACO) with DSR called Enhanced Ant DSR. This technique uses ACO to select the optimal path. The pheromone count (probability to choose a path) of the route is calculated taking into account the Link Metric (LM), Congestion Metric (CM), and Average hop counts.

In 2016 there was research related to QoS (Quality of Service)-Aware by utilizing the Ant colony Algorithm. Al-Ani \& Seitz (2016) This research proposed Routing-aware QoS based on Ant Colony Optimization (QoRA). QoRA calculates the QoS parameters locally and avoids congestion during data transmission with the help of two architectural components namely congestion and SNMP. MANET research on the AODV reactive protocol with the development of the K-Means cluster method has been carried out in an article published in 2016. Shirazi \& Mirabedini (2016) In this research, a dynamic K -means algorithm is presented to improve the routing process in the Mobile Ad-Hoc network (MANETs). The changes that occur due to movement are tried to be overcome by adding a cluster feature.

Besides, this research also tries to solve various problems in the routing process such as increased message overhead and inefficient routing between network nodes.

In this research, the development of the AODV protocol was carried out by adding the K-Means and Ant Colony clustering methods. The performance of the development carried out is measured, hope that the overall network performance can increase. The main objective is to increase the Quality of Service (QoS) of the MANET network as measured by the parameters of Packet Delivery Ratio (PDR), End to End Delay (E2E), and also Average Hop Count (AHC). Simulations were carried out with the Network Simulator NS2.35 in the Linux operating system environment. Routing protocol studied was Ad-hoc On Demand Vector (AODV) developed with Ant-Colony and K-Means Clustering. Number of nodes and clusters is also limited according to the parameters.

The area on this research has an area of 900 meters square with a real random waypoint movement. The simulation time is 200 seconds with simulation experiments with 10,15 , and 20 clusters, respectively. The number of nodes that are run is $100,150,200,250$, and 300 nodes. Using the Random Waypoint mobility model with an initial energy of 150 joules, a transmission radius of 400 meters. Mac Protocol IEEE 802.11 with Wireless channel type.

\section{MATERIALS AND METHODS}

This research begins with a modification by adding the K-Means Clustering method. Clustering is carried out at a predetermined time when the simulation starts. In other words, it is necessary to send a Hello Message to map the location of neighboring nodes and after getting the position, the Clustering process is carried out based on the data that has been obtained. The result of clustering nodes is some n-clusters where each cluster will get a Cluster Head and Cluster Gateway. See Figure 1 for details of the development process carried out

Each Cluster will only send Route Request RREQ through the Cluster Head and Cluster Gateway. After RREQ is sent, the next process is to find the best route using Ant-Colony. The antcolony here has been modified so that it has a pheromone value parameter that has been adjusted to be stored during the route search process.

The pheromone value will be added as the route search is carried out through each node. When it arrives at the Destination Node, the total pheromone value will be returned through the RREP route reply process until it reaches the Source Node. The pheromone values inserted in the RREP will be compared at the Source Node to get the best route with the largest pheromone value. In the section on creating the Mobility Flow and Activity Flow from Node, it is designed using the tools available in Network Simulator software or NS version 2.35.

In this simulation, nodes move with the random waypoint mobility model. Nodes are randomly distributed in the simulation area. Each node uses an omnidirectional antenna which has a range of 250 meters. Each node moves at a predetermined speed. Traffic will start at a random time from the start of the simulation at random and remain active throughout the simulation. Source node generates constant bit rate (CBR) traffic during the simulation. 




Figure 1. Flowchart of AODV Routing Protocol with Ant-Colony and K-Means Clustering

To generate a random movement of the Mobility Random Waypoint model, in this study a tool known as a setdest was used. Meanwhile, the tools to generate a random data communication model used the familiar cbrgen tools in NS 2.35. Referring to the research references that we use, these two tools are widely used in evaluating the performance of MANET network algorithms and protocols. The Random Waypoint mobility model used in this simulation is emphasized according to the reference which informs the similarity of the behavior of the movement patterns of wireless network users who form the MANET network (Pramanik, Choudhury, Choudhury, Arif, \& Mehedi, 2015).

\section{RESULT AND DISCUSSION}

Analysis of the results and evaluation of the simulation is done by processing the results of the trace file of the simulation that was carried out. The parameters analyzed according to the test results are the packet delivery ratio, end to end delay, routing overhead, and also the Average Hop Count. Analysis of the results of the trace file is carried out with the AWK script, which is commonly used to analyze the results of simulations that have been successfully run.

Packet Delivery Ratio (PDR) is the ratio between the number of packets received and the number of packets sent. To calculate the packet delivery ratio, the following calculation formula is used:

$$
P D R=\frac{\text { Packet Received }}{\text { Packet Sent }} \times 100 \%
$$

PDR improved value is expressed as a percentage that states the success of the data packet sent. PDR high percentage value represents the successful delivery of packets sent.

Improved packet delivery ratio (PDR) on

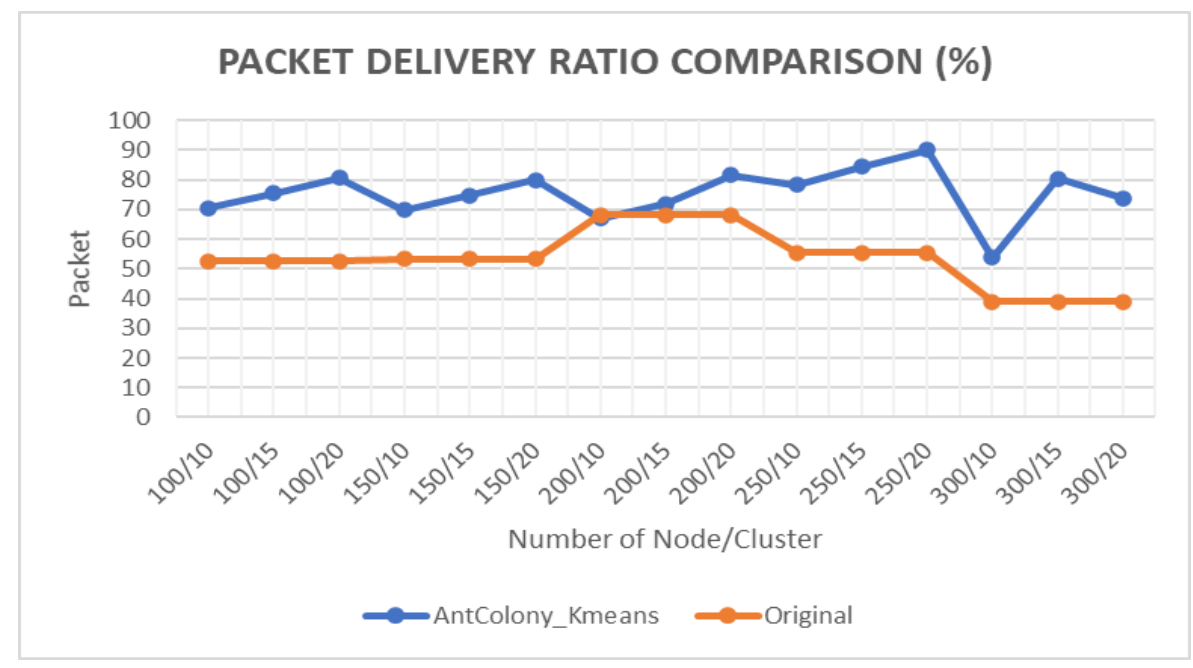

Figure 2. Comparison of PDR modified and original AODV simulation results 
Journal Of Development Research, 5 (1), May 2021, Pages 1-6

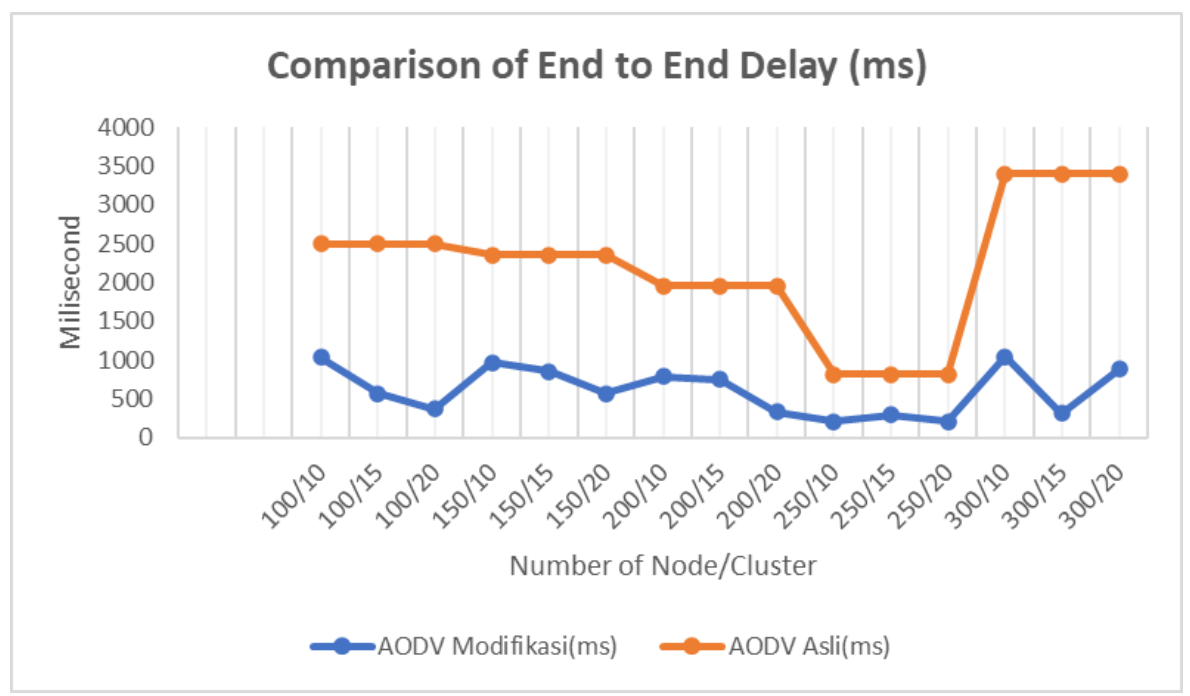

Figure 3. Comparison of E2D modified and original AODV simulation results

the modification of the addition of Ant-Colony and K-Means Clustering occurs on all models Cluster. PDR decrease occurs when the simulation runs on the number of 200 Nodes with a 10 cluster model. The increased packet delivery ratio in modified AODV was due to better search for delivery routes. Calculation of pheromones by considering various factors and also the formation of clusters currently shipping shown to increase the success of packets transmitted. Increasing best PDR occurred in the simulation with the value of 15 on a 300 node cluster with an increase of $41.59 \%$. Packet Delivery Ratio Comparison can be seen on Figure 2.

End to End Delay (E2D) is the average of the delay or time it takes from a packet sent to the destination node in seconds. The delay value is obtained from the period required from the packet sent by the source node to the destination node. The total delay for all packages is then calculated and divided by the number of packages that have arrived or were successfully received.

To calculate the end to end delay, you can use the following formula:

$$
E 2 D=\frac{\sum_{\text {issent }}^{i=0}\left(t_{\text {received }[i]}-t_{\text {sent }[i]}\right)}{\text { sent }}
$$

E2D is rated in milliseconds (ms), $\mathrm{i}$ is the sequence/id of the i-packet. While t-received is the time the packet is received in milliseconds (ms), as well as t-sent which is the time when the packet is sent in milliseconds (ms). Sent here is the number of data packets sent.

E2D measurement results are measured by the simulation results carried out by the End to End Delay measurement parameter in milliseconds. It can be seen from the development results that it reduces the overall delay in the run simulation. End to End delay the average from simulation can collect as much as $319.42 \%$. As the number of nodes increases, the original AODV manages to reduce the delay, but by adding Ant Colony and K-Means clustering the delay can gather low. See Figure 3. for the detail of E2D comparison. As the number of nodes increases, AODV maintains network connectivity by improving route changes. However, as the nodes increase, as the mobility of the nodes increases, there is an increase in delay. With the method of developing Ant Colony and K-Means clustering, this can continue to grow. Some of the studies that have been referred to also have fluctuating graphic trends. Selection of the Random Way Point scenario that describes the real scheme that results from different results in simulation measurements (Pramanik et al., 2015).

Average Hop Count is the average number of nodes passed by each packet sent from the source node to the destination node. A modified AODV should have an average hop count less than the original AODV. Average Hop Count can be calculated using the formula:

$$
\text { Average Hop Count }=\frac{\sum_{n=1}^{\text {recvpackt }} \text { hop count }}{\text { recvpackt }}
$$




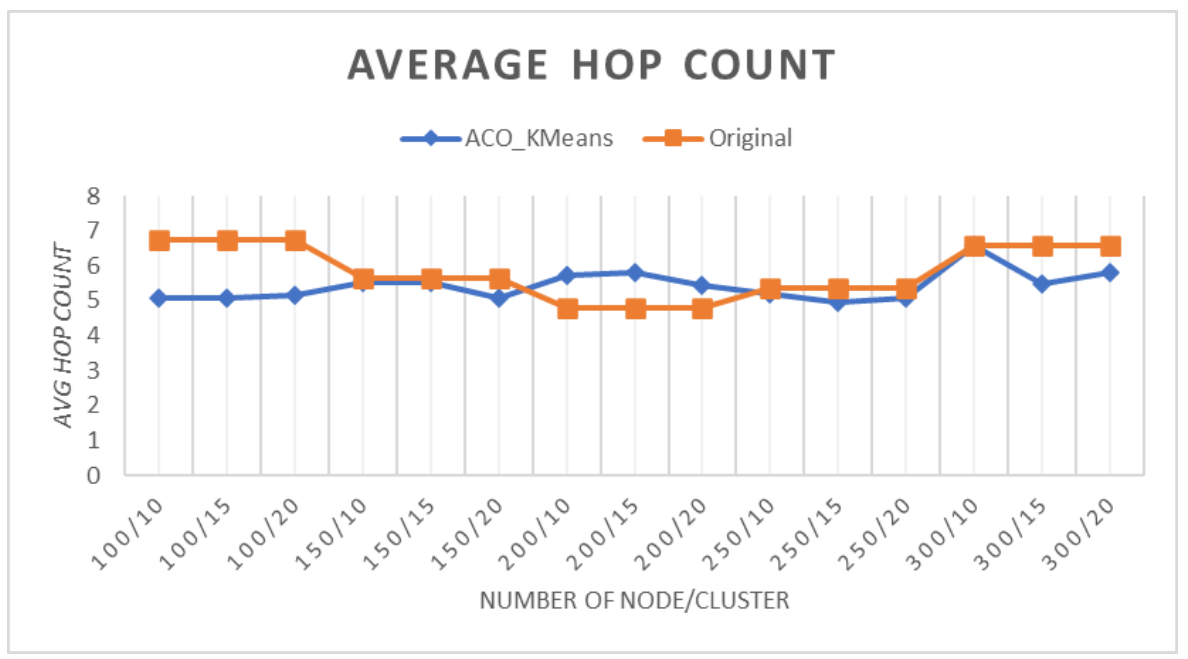

Figure 4. Comparison of AHC modified and original AODV simulation results

Wherefrom this formula, recvpackt is the number of packets that were successfully sent to the destination node. While the hop count is the number of jumps from the packet through the intermediary node until it reaches its destination.

As it can be seen from the Figure 4. that the Average Hop Count of the development results fluctuates in several simulation model schemes that are run. In general, the developments that have been carried out have succeeded in reducing the Average Hop Count measurement by $7.25 \%$. Even though the 200 node scheme is not better at suppressing the Average Hop Count value. This is because the measurement of the Ant-Colony pheromone value at the 200 node simulation has changed and affects the $\mathrm{K}-\mathrm{Means}$ cluster method that has been made. So that the hop count in packet delivery should be suppressed. However, because the mobility of the nodes resulted in changing pheromone measurement parameters so that overall in the 200 node simulation, the combination of the two methods could not effectively reduce the hop count.

\section{Conclusion}

By making modifications to the development of the Ant-Colony method combined with the K-Means clustering method, it has succeeded in increasing the performance of AODV on the MANET. The performance of the development results that have been carried out has increased significantly. This is evidenced by in- creasing the value of the Packet delivery ratio, reducing the End to End Delay and Average Hop Count. Our suggestion for future research that is necessary to solve the problem that causes the fluctuative trend value by adding other methods. Application to a more varied simulation can be done to get more accurate results. Reducing the assessment parameters in the Ant-Colony method can be done with the hope that the fewer parameters measured can reduce the Routing Overhead.

\section{REFERENCES}

Chatterjee, S., \& Das, S. (2015). Ant colony optimization based enhanced dynamic source routing algorithm for mobile Ad-hoc network. Information Sciences, 295, 67-90. https:// doi.org/10.1016/j.ins.2014.09.039

Pramanik, A., Choudhury, B., Choudhury, T. S., Arif, W., \& Mehedi, J. (2015). Simulative study of random waypoint mobility model for mobile ad hoc networks. In Global Conference on Communication Technologies, GCCT 2015 (pp. 112-116). Institute of Electrical and Electronics Engineers Inc. https://doi.org/10.1109/ GCCT.2015.7342634

Sharma, A., \& Kumar, R. (2016). Performance comparison and detailed study of AODV, DSDV, DSR, TORA and OLSR routing protocols in ad hoc networks. In 2016 Fourth International Conference on Parallel, Distributed and Grid Computing (PDGC) (pp. 732-736). IEEE. https://doi.org/10.1109/ PDGC.2016.7913218

Shirazi, Z. Z., \& Mirabedini, S. J. (2016). Dy- 
namic K-Means Algorithm for Optimized Routing in Mobile Ad Hoc Networks. International Journal of Computer Science \& Engineering Survey, 7(2), 01-14. https:// doi.org/10.5121/ijcses.2016.7201

Singh, M., \& Kumar, S. (2017). A Survey: Adhoc on Demand Distance Vector (AODV) Protocol. International Journal of Computer Applications, 161(1), 38-44. https:// doi.org/10.5120/ijca2017913109

Wang, J., Osagie, E., Thulasiraman, P., \& Thulasiram, R. K. (2009). HOPNET: A hybrid ant colony optimization routing algorithm for mobile ad hoc network. Ad Hoc Networks, 7 (4), 690-705. https://doi.org/10.1016/ j.adhoc.2008.06.001 